\title{
Clinical Study \\ Bacteremia after Endoscopic Submucosal Excavation for Treating the Gastric Muscular Layer Tumors
}

\author{
Guohua Li, Sheng Zeng, Youxiang Chen, Xiaojiang Zhou, and Nonghua Lv \\ Department of Gastroenterology, The First Affiliated Hospital of Nanchang University, Nanchang, Jiangxi 330006, China \\ Correspondence should be addressed to Nonghua Lv; lunonghua@163.com
}

Received 7 December 2014; Accepted 23 March 2015

Academic Editor: Lana Bijelic

Copyright ( 2015 Guohua Li et al. This is an open access article distributed under the Creative Commons Attribution License, which permits unrestricted use, distribution, and reproduction in any medium, provided the original work is properly cited.

\begin{abstract}
Background. The bacteremia is reported as being infrequent and transient in gastric EMR and ESD for treating gastric mucosa lesions or superficial gastric neoplastic lesion. There was no report of it being investigated in ESD for treating gastric muscular layer tumors (endoscopic submucosal excavation, ESE). This study aimed to determine the frequency of bacteremia in gastric ESE. Patients and Methods. A prospective study, in 122 consecutive patients who underwent gastric ESE for treating gastric muscular layer tumors, investigated the frequency of bacteremia before and 15 minutes after the procedure. Results. The median time for the total ESE procedure was $29 \mathrm{~min}$ (range from 8 to $62 \mathrm{~min}$ ). The mean size of the biggest diameter of each resected specimen was $10 \pm 2.7 \mathrm{~mm}$ (range from $5 \mathrm{~mm}$ to $30 \mathrm{~mm}$ ). Blood cultures obtained before ESE were positive in 0\% (0/122) of cases. Blood cultures obtained 15 min after ESE were positive in 2.5\% (3/122) of cases. Six blood samples contained Staphylococcus with coagulase negative, which was considered contaminant. No signs of sepsis were seen in all patients. Conclusions. The frequency of bacteremia after gastric ESE was low. ESE for treating gastric lesions is thought to have a low risk of infectious complications; therefore, prophylactic administration of antibiotics may not be warranted.
\end{abstract}

\section{Introduction}

Bacteremia may develop endocarditis or other infection complications. As a result, the American Society for Gastrointestinal Endoscopy (ASGE), the American Heart Association (AHA), and other societies recommended prophylactic administration of antibiotics for high-risk patients undergoing high-risk procedures [1-6]. It has been known that there was an increased rate of bacteremia or local infection after some endoscopy procedures (such as esophageal sclerotherapy, esophageal stricture dilation, endoscopic retrograde cholangiopancreatography with biliary obstruction, endoscopic drainage of a pancreatic pseudocyst, endoscopic placement of feeding tubes, and EUS-FNA) [7-20]. So those guidelines recommended some endoscopy procedures should use prophylactically antibiotics in some high-risk patients. Until now, there were no formal recommendations regarding the need for prophylactic antibiotic administration in patients undergoing endoscopic submucosal dissection and endoscopic submucosal excavation. The aim of this study was to prospectively evaluate the frequency of bacteremia and associated complications after endoscopic submucosal excavation.

\section{Patients and Methods}

One hundred and twenty-two consecutive patients with gastric muscular layer tumor enrolled in our study (60 women, 62 men; median age 45 years, range 22 to 74 years). All patients signed informed consent before the procedure. Exclusion criteria were the following: (1) bacterial infection and/or antibiotic treatment within 3 weeks; (2) need for antibiotic prophylaxis according to the American Heart Association Guidelines; (3) immune deficiency status; (4) age less than 18 years; (5) occurrence of contraindications for endoscopy procedures; (6) perforation of gastric wall during ESE procedure; and (7) lack of informed consent. This study was approved by the ethics committee of our hospital.

The indications for ESE were the following: (1) the tumor located in the gastric muscular layer; (2) the diameter of mass ranged from $5 \mathrm{~mm}$ to $30 \mathrm{~mm}$; and (3) the mass showed hypoecho under ultrasonic endoscopy. 
TABle 1: The positive blood culture results.

\begin{tabular}{|c|c|c|c|c|c|}
\hline Number & Location of the tumor & Before ESE & Fifteen minutes after ESE & Operation time & Size $(\mathrm{cm})$ \\
\hline Number 1 & Fundus & Negative & Staphylococcus capitis & $28 \mathrm{~min}$ & 0.6 \\
\hline Number 2 & Body of stomach & Negative & Staphylococcus capitis & $35 \mathrm{~min}$ & 1.3 \\
\hline Number 3 & Fundus & Negative & Staphylococcus warmen & $34 \mathrm{~min}$ & 1.2 \\
\hline Number 4 & Fundus & Negative & Staphylococcus warmen & $28 \mathrm{~min}$ & 0.9 \\
\hline Number 5 & Body of stomach & Negative & Staphylococcus hominis & $29 \mathrm{~min}$ & 2.5 \\
\hline Number 6 & Body of stomach & Negative & Staphylococcus cohnii & $31 \mathrm{~min}$ & 1.0 \\
\hline Number 7 & Fundus & Negative & Gram positive bacilli & $32 \mathrm{~min}$ & 0.5 \\
\hline Number 8 & Body of stomach & Negative & Gram positive bacilli & $29 \mathrm{~min}$ & 0.8 \\
\hline Number 9 & Body of stomach & Negative & Micrococcus luteus & $31 \mathrm{~min}$ & 0.8 \\
\hline
\end{tabular}

\section{Microbiologic Examination}

Immediately before and 15 minutes after ESE, $12 \mathrm{~mL}$ of blood was drawn by separate peripheral venipuncture by the same examiner. The specimens were equally distributed into an aerobic blood culture bottle and an anaerobic blood culture bottle (BAcT/ALERT 3D). Venipuncture was performed under a germ-free condition after the skin was sterilized with povidone-iodine and left at least 30 seconds for bactericidal effect. Blood cultures were incubated at $35^{\circ} \mathrm{C}$ for 5 days. If there was bacterial growth, the microorganisms were identified and tested for antibiotic sensitivity. All patients with a positive culture result had observed clinical signs of infection for at least 5 further days.

\section{ESE Procedure and Treatment after ESE}

Prior to an endoscopic submucosal excavation, all lesions were confirmed to be originated from muscularis propria by EUS (GF-UC240P-AL5, Olympus). Endoscopic submucosal excavation procedure was performed under intravenous anesthesia with propofol (GF-UC240P-AL5, Olympus) according to the following steps. The superficial mucosa of the lesion was marked with APC ( $40 \mathrm{~W}$ soft coagulation) to determine the location of the tumor. A salt solution containing $0.005 \mathrm{mg} / \mathrm{mL}$ epinephrine and $0.1 \%$ indigo carmine was injected into the submucosa. After sufficient lifting, a hook knife (KD 620LR, Olympus) was used to cut the superficial mucosa open along a straight line. The separation of the submucosal layer and the tumor was performed carefully with IT knife-2 (KD 611L, Olympus) under the endocut mode of electrosurgical accessories (ICC300, Erbe Co.). To avoid bleeding, small vessels were coagulated directly by knives; large vessels with high bleeding risk were coagulated with hemostatic forceps (Olympus). The body of the tumor was gradually exposed when the incision was wide enough. The muscularis propria tumor was snared or continued to be dissected by the IT knife after complete exposure of the root of the tumor. After resection of the tumor, the incision was closed by clips. The stomach tube was inserted into stomach for at least $24 \mathrm{~h}$. The specimen was collected and sent to pathology department. The patients received transfusion and PPI after being transported to ward. The patients fasted for at least $24 \mathrm{~h}$ and then removed the stomach tube and took liquid or semifluid for 7 days. No symptoms and signs were observed in all patients for at least the following 7 days.

\section{Results}

All tumors that originated from muscularis propria were removed from 122 patients. The median size was $10 \pm 2.7 \mathrm{~mm}$ (range from $5 \mathrm{~mm}$ to $30 \mathrm{~mm}$ ). The median operation time was $29 \pm 9.4 \mathrm{~min}$ (range from $8 \mathrm{~min}$ to $62 \mathrm{~min}$ ). The locations of the tumors in fundus, body of stomach, and antrum were $24.6 \%$ (30/122), 65.6\% (80/122), 9.8\% (12/122), respectively. There were 83 stromal tumors, 37 leiomyomas, and 2 neurinomas. All patients had no symptoms and signs of peritonitis.

The blood cultures of 244 samples from 122 patients were performed before and $15 \mathrm{~min}$ after ESE. There were nine positive culture samples from 9 patients' blood samples 15 minutes after ESE. The results are listed in Table 1. Among nine positive blood culture samples, there were four kinds of Staphylococcus with coagulase negative from six samples. The six positive blood cultures were considered to be contaminant. There were two samples with gram positive bacilli and one sample with Micrococcus luteus. All patients remained asymptomatic without fever (defined as an oral temperature of $38^{\circ} \mathrm{C}$ ) or chills during the observation period in gastroenterology department. There was no infectious complication (e.g., fever, phlegmon, or abscess formation) reported by any subjects or the referring physicians at one week after ESE.

\section{Discussion}

In daily living, transient bacterial seeding of the blood commonly may appear while brushing tooth and chewing food [20]. Bacteremia develops also commonly after some operation (e.g., nasogastric intubation, digital rectal examination, barium enema, and uncomplicated vaginal delivery) [20-25]. Antibiotic prophylaxis is clearly not indicated in these settings because of the limited duration (less than 15 minutes) of bacteremia and lack of clinical sequelae.

The longer time bacteremia can lead to bacterial endocarditis in some high-risk or moderate-risk patients. The AHA and ASGE recommended prophylactic administration of antibiotics for patients with high-risk cardiac conditions who are undergoing high-risk procedures [1-3]. 
Until recently, there were rare studies about bacteremia incidence after ESD [26-28]. Itaba et al. [26] reported that the bacteremia incidence was $4.4 \%(2 / 45)$ before gastric ESD, $4.3 \%(2 / 46) 10 \mathrm{~min}$ after gastric ESD, and $0 \%(0 / 46) 3 \mathrm{~h}$ after gastric ESD. Kato et al. [27] reported that the bacteremia incidence was $2 \%(2 / 100)$ after gastric ESD. So the bacteremia incidence may be low and transient after gastric ESD. In their studies, most gastric lesions originated from mucosa layer or submucosa layer; fewer lesions originated from muscular layer. But there was no study about the bacteremia incidence after ESE for gastric muscularis propria tumors. This study researched prospectively the bacteremia incidence after ESE for gastric muscularis propria tumors.

In the present study, bacteremia developed in three patients $(2.5 \%, 3 / 122) 15$ minutes after ESE. Six patients grew coagulase-negative Staphylococcus $(n=6)$, which deemed contaminants, not typically associated with ESE. All samples grew no bacteria before ESE. There was no evidence of immediate or delayed infection complications in any patients with positive blood cultures (actual or contaminant) after ESE. So bacteremia incidence was low after ESE and may be transient because of all patients without infection complications. Why coagulase-negative Staphylococcus was taken for contaminant bacteria in this paper? The coagulasenegative Staphylococcus is the most common flora in skin, and the most common contaminant bacteria in blood culture. There is a few coagulase-negative Staphylococcus in upper gastrointestinal tract of normal human. It is scarcely possible that endoscope was contaminated by coagulasenegative Staphylococcus in skin because the endoscope was sterilized by orthophthalaldehyde before ESE, and the operator abided by aseptic technique during procedure. It is also scarcely possible that the endoscope was contaminated by upper gastrointestinal tract flora because the blood culture was single coagulase-negative Staphylococcus in the 6 patients. Moreover, the six patients with coagulase-negative Staphylococcus blood culture had not any infection symptoms. Thereby we took coagulase-negative Staphylococcus for contaminant bacteria as other studies [26, 27].

The operation time and size of tumor may affect the bacteremia incidence after gastric ESE. In the present study, the diameter of tumor ranged from $5 \mathrm{~mm}$ to $30 \mathrm{~mm}$. We select those sizes of tumors to treat for the following reasons. (1) The most muscularis propria tumor of stomach was stromal tumor. Malignant degree may increase if the diameter more than $30 \mathrm{~mm}$, which may be not proper to remove by ESE. (2) The ESE for treating these tumors might have infrequent complication and less operation time. The median operation time was $29 \mathrm{~min}$ (from $8 \mathrm{~min}$ to $62 \mathrm{~min}$ ). So it was safe and efficient that endoscopic excavation of these size tumors. In the patients with positive blood culture, the median operation time was $31 \mathrm{~min}$ (from $28 \mathrm{~min}$ to $35 \mathrm{~min}$ ), and mean size was $10.7 \mathrm{~mm}$. The median operation time and the mean size of patients with positive blood culture were similar to those in patients with negative blood culture. So the positive blood cultures were not related to tumor size (less than $30 \mathrm{~mm}$ ) and operation time.

The perforation of stomach may affect the bacteremia incidence during ESE procedure. The abdominal cavity of those patients with perforation may be contaminated by gastrointestinal bacteria. These patients should administrate prophylactic antibiotics. So we excluded these patients with perforation in our study.

In summary, bacteremia develops after ESE of gastric muscularis propria tumors (less than $30 \mathrm{~mm}$ ) at a low rate similar to that for diagnostic endoscopy examination. ESE of gastric muscularis propria tumors (less than $30 \mathrm{~mm}$ ) should be considered a low-risk procedure for infection complications, which does not warrant the prophylactic administration of antibiotics for the prevention of endocarditis.

\section{Conflict of Interests}

The authors declare that there is no conflict of interests regarding the publication of this paper.

\section{Acknowledgments}

This study was supported by the First Affiliated Hospital of Nanchang University and authorized by the hospital ethics committee.

\section{References}

[1] N. Rahman, S. Rogers, D. Ryan, C. Healy, and S. Flint, "Infective endocarditis prophylaxis and the current AHA, BSAC, NICE and Australian guidelines," Journal of the Irish Dental Association, vol. 54, no. 6, pp. 264-270, 2008-2009.

[2] P. van der Bijl, "Antibiotic prophylaxis for infective endocarditis: new AHA and BSAC guidelines and implications for practice in South Africa," SADJ, vol. 63, no. 4, pp. 240-244, 2008.

[3] S. Banerjee, B. Shen, T. H. Baron et al., "Antibiotic prophylaxis for GI endoscopy," Gastrointestinal Endoscopy, vol. 67, no. 6, pp. 791-798, 2008.

[4] J. Snyder and B. Bratton, "Antimicrobial prophylaxis for gastrointestinal procedures: current practices in North American academic pediatric programs," Journal of Pediatric Gastroenterology and Nutrition, vol. 35, no. 4, pp. 564-569, 2002.

[5] "Infection control during gastrointestinal endoscopy: guidelines for clinical application. From the ASGE. American Society for Gastrointestinal Endoscopy," Gastrointestinal Endoscopy, vol. 49, no. 6, pp. 836-841, 1999.

[6] A. S. Dajani, K. A. Taubert, W. Wilson et al., "Prevention of bacterial endocarditis: recommendations by the American Heart Association," Clinical Infectious Diseases, vol. 25, no. 6, pp. 1448-1458, 1997.

[7] S. G. Kulkarni, S. S. Parikh, P. S. Dhawan et al., "High frequency of bacteremia with endoscopic treatment of esophageal varices in advanced cirrhosis," Indian Journal of Gastroenterology, vol. 18, no. 4, pp. 143-145, 1999.

[8] E. B. Maulaz, A. A. de Mattos, J. Pereira-Lima, and J. Dietz, "Bacteremia in cirrhotic patients submitted to endoscopic band ligation of esophageal varices," Arquivos de Gastroenterologia, vol. 40, no. 3, pp. 166-172, 2003.

[9] R. Rerknimitr, J. Chanyaswad, P. Kongkam, and P. Kullavanijaya, "Risk of bacteremia in bleeding and nonbleeding gastric varices after endoscopic injection of cyanoacrylate," Endoscopy, vol. 40, no. 8, pp. 644-649, 2008. 
[10] D. Q. Bonilhai, L. M. Correia, M. Monaghan, L. Lenz, M. Santos, and E. D. Libera, "Prospective study of bacteremia rate after elective band ligation and sclerotherapy with cyanoacrylate for esophageal varices in patients with advanced liver disease," Arquivos de Gastroenterologia, vol. 48, no. 4, pp. 248-251, 2011.

[11] M. Barawi, K. Gottlieb, B. Cunha, M. Portis, and F. Gress, "A prospective evaluation of the incidence of bacteremia associated with EUS-guided fine-needle aspiration," Gastrointestinal Endoscopy, vol. 53, no. 2, pp. 189-192, 2001.

[12] J. Janssen, K. König, V. Knop-Hammad, W. Johanns, and L. Greiner, "Frequency of bacteremia after linear EUS of the upper GI tract with and without FNA," Gastrointestinal Endoscopy, vol. 59, no. 3, pp. 339-344, 2004.

[13] M. J. Levy, I. D. Norton, M. J. Wiersema et al., "Prospective risk assessment of bacteremia and other infectious complications in patients undergoing EUS-guided FNA," Gastrointestinal Endoscopy, vol. 57, no. 6, pp. 672-678, 2003.

[14] M. J. Levy, I. D. Norton, J. E. Clain et al., "Prospective study of bacteremia and complications with EUS FNA of rectal and perirectal lesions," Clinical Gastroenterology and Hepatology, vol. 5, no. 6, pp. 684-689, 2007.

[15] C. Guarner-Argente, P. Shah, A. Buchner, N. A. Ahmad, M. L. Kochman, and G. G. Ginsberg, "Use of antimicrobials for EUSguided FNA of pancreatic cysts: a retrospective, comparative analysis," Gastrointestinal Endoscopy, vol. 74, no. 1, pp. 81-86, 2011.

[16] W. C. Chen, M. C. Hou, H. C. Lin et al., "Bacteremia after endoscopic injection of N-butyl-2-cyanoacrylate for gastric variceal bleeding," Gastrointestinal Endoscopy, vol. 54, no. 2, pp. 214-218, 2001.

[17] D. B. Nelson, S. J. Sanderson, and M. M. Azar, "Bacteremia with esophageal dilation," Gastrointestinal Endoscopy, vol. 48, no. 6, pp. 563-567, 1998.

[18] M. Brand, D. Bizos, and P. O’Farrell Jr., "Antibiotic prophylaxis for patients undergoing elective endoscopic retrograde cholangiopancreatography," Cochrane Database of Systematic Reviews, no. 10, Article ID CD007345, 2010.

[19] J. Llach, J. M. Bordas, M. Almela et al., "Prospective assessment of the role of antibiotic prophylaxis in ERCP," HepatoGastroenterology, vol. 53, no. 70, pp. 540-542, 2006.

[20] E. D. Everett and J. V. Hirschmann, "Transient bacteremia and endocarditis prophylaxis. A review," Medicine, vol. 56, no. 1, pp. 61-77, 1977.

[21] J. M. B. Casas, J. M. H. Hernández, M. A. G. Jiménez et al., "Bacteremia caused by digestive system endoscopy," Revista Española de Enfermedades Digestivas, vol. 91, no. 2, pp. 105-116, 1999.

[22] D. Franchi, A. Bahrani, J. F. Ober, and M. B. Edmond, "Preventing nosocomial infections from gastrointestinal endoscopy," Current gastroenterology reports, vol. 2, no. 4, pp. 294-298, 2000.

[23] D. B. Nelson, "Infection control during gastrointestinal endoscopy," Journal of Laboratory and Clinical Medicine, vol. 141, no. 3, pp. 159-167, 2003.

[24] B. C. Cho, J. H. Lee, J. W. Park et al., "Subacute bacterial endocarditis associated with upper endoscopy," Yonsei Medical Journal, vol. 45, no. 5, pp. 936-940, 2004.

[25] K. R. Jones, C. W. Maddox, M. D. Ridgway, S. C. ClarkPrice, and O. Dossin, "Incidence of bacteremia following upper gastrointestinal endoscopy and biopsy in healthy dogs before, during, and after treatment with omeprazole," American Journal of Veterinary Research, vol. 74, no. 2, pp. 239-242, 2013.
[26] S. Itaba, Y. Iboshi, K. Nakamura et al., "Low-frequency of bacteremia after endoscopic submucosal dissection of the stomach," Digestive Endoscopy, vol. 23, no. 1, pp. 69-72, 2011.

[27] M. Kato, M. Kaise, T. Obata et al., "Bacteremia and endotoxemia after endoscopic submucosal dissection for gastric neoplasia: pilot study," Gastric Cancer, vol. 15, no. 1, pp. 15-20, 2012.

[28] B. H. Min, D. K. Chang, D. U. Kim et al., "Low frequency of bacteremia after an endoscopic resection for large colorectal tumors in spite of extensive submucosal exposure," Gastrointestinal Endoscopy, vol. 68, no. 1, pp. 105-110, 2008. 


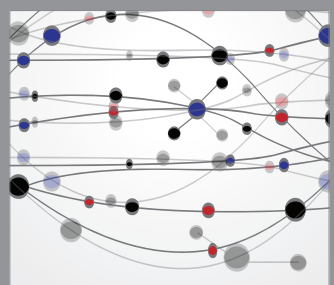

The Scientific World Journal
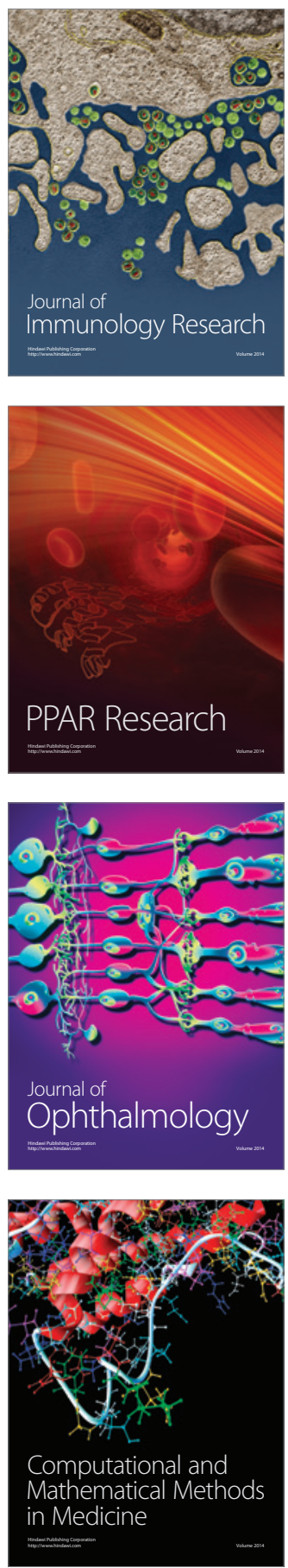

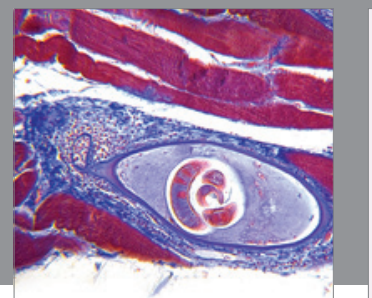

Gastroenterology

Research and Practice
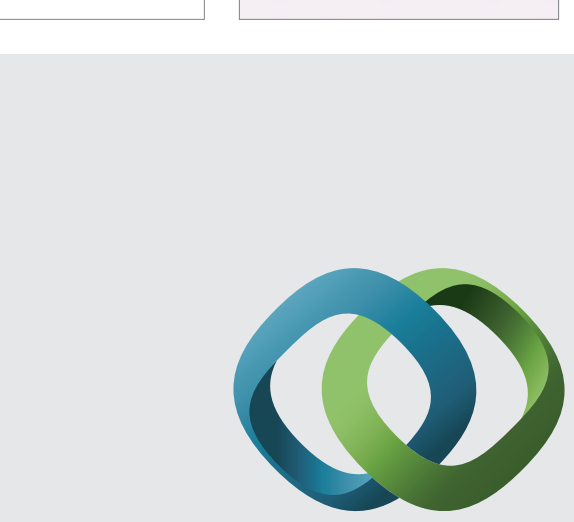

\section{Hindawi}

Submit your manuscripts at

http://www.hindawi.com
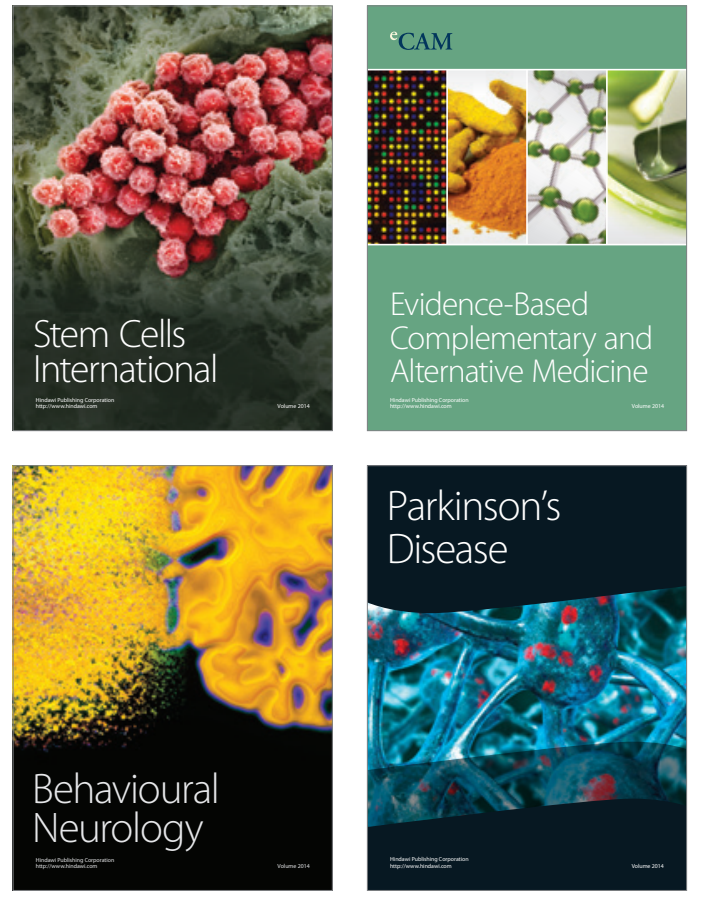
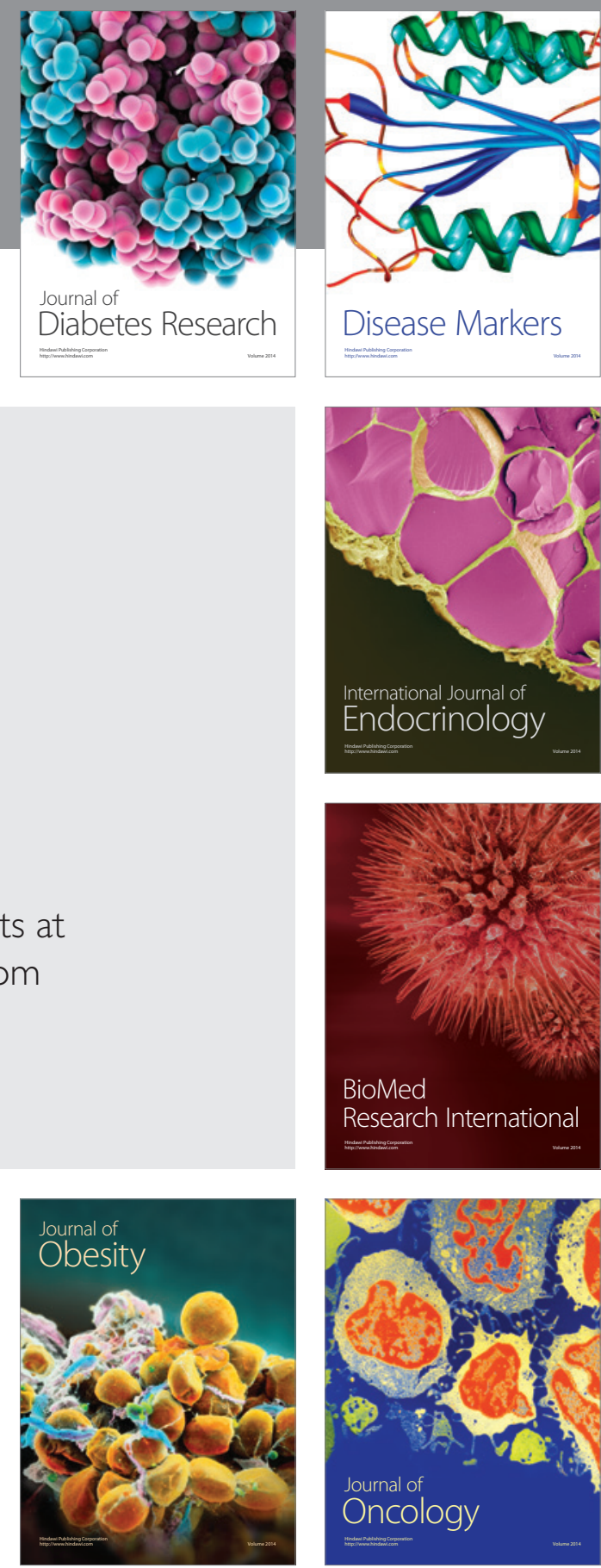

Disease Markers
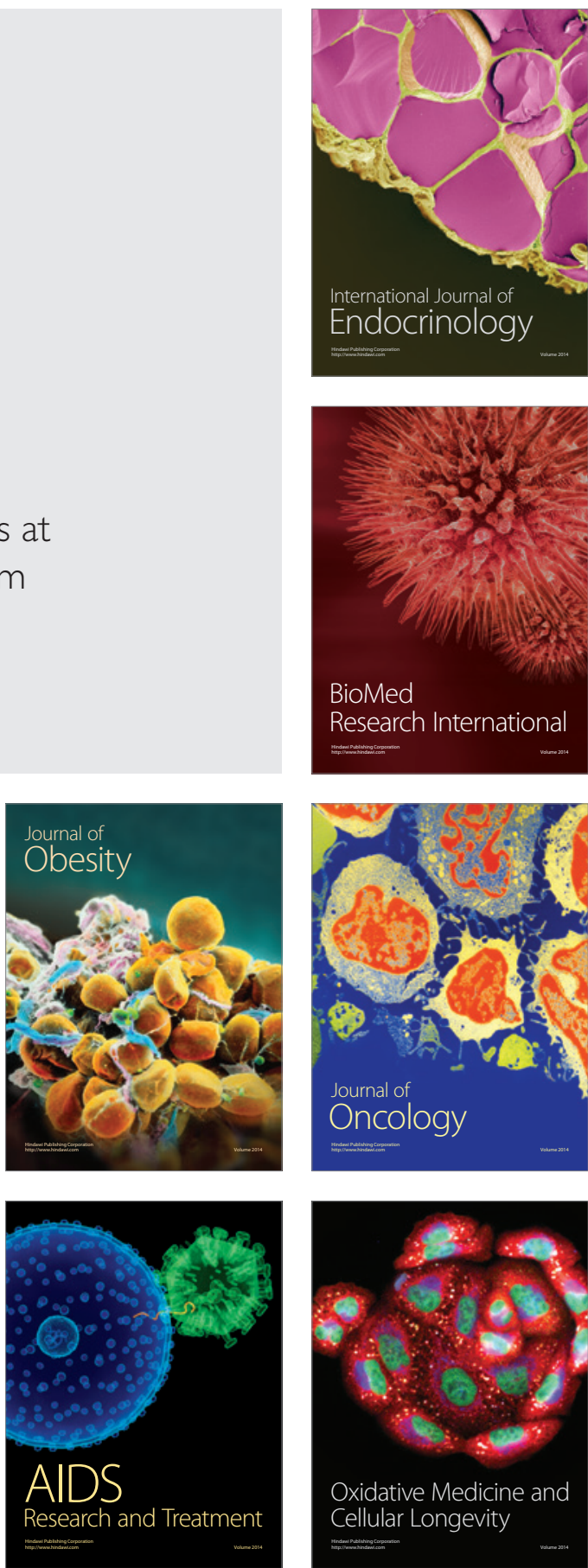